Pacific Journal of Mathematics

LINEAR FUNCTIONAL EQUATIONS AND INTERPOLATION 


\title{
LINEAR FUNCTIONAL EQUATIONS AND INTERPOLATION SERIES
}

\author{
Philip DaVis
}

1. Introduction. The question of obtaining complete sets of solutions for a given linear partial differential equation is of the greatest interest from the theoretical as well as from the computational point of view. For constructing such sets, several methods of considerable generality have been proposed. Thus, for instance, Bergman [3] has introduced an integral operator which provides a means for the generation of complete sets when the differential equation is of the second or the fourth order. Extensions may be made to higher orders. By means of Bergman's operator, the space of analytic functions of a single complex variable is mapped upon the space of solutions of the given differential equation, and the process yields a generalization of the operator $R e$ in the case of harmonic functions.

Complete sets of solutions may also be found by a method which is analogous to Runge's method of approximation in the theory of analytic functions. A description of this may be found in [6, p.282]. This scheme has the practical drawback of requiring a knowledge of a fundamental singularity for the differential equation, a function which is known explicitly for but few differential equations.

In the present paper, we adopt a different point of view and study possible representations of solutions of linear functional equations of a certain class, and the generation of complete sets of such solutions by means of generalized interpolation series. By this is meant a biorthogonal series of the form

$$
f \sim \sum_{n=0}^{\infty} L_{n}(f) \phi_{n} ; \quad L_{m}\left(\phi_{n}\right)=\delta_{m n}
$$

Here $\left\{L_{n}\right\}$ is a sequence of linear functionals. When each $L_{n}$ is a point or a linear differential operator, then the series (1) reduces to a classical interpolation series. Our method is, essentially, to reduce the problem of the solution of the linear functional equation to a problem involving a denumerable infinity

Received May 7, 1953. This work was performed under a National Bureau of Siandards contract with American University, and was sponsored by the Wright Air Development Center, ARDC, USAF.

Pacific J. Math. 4 (1954), $503-532$ 
of interpolatory conditions. An interpolatory procedure then yields an operator which may be cast into integral form, and which maps an appropriate space of functions onto a subspace of solutions.

In order to carry out this method with ease, it is convenient to deal with Hilbert spaces $H$ of functions, $H$ being supposed to possess a reproducing kernel $[\mathrm{cf} .5, \mathbf{1}]$, to restrict our basic functional equations to those possessing certain boundedness properties with respect to $H$, and to consider only solutions which lie in $H$. These assumptions will cause no difficulty in many instances where the existence and regularity of solutions may be known beforehand from independent considerations. Our work, therefore, falls mainly within the region of representation theory.

It is our principal aim to construct interpolation series which converge in preassigned regions to solutions of linear functional equations, and, by way of corollary, to construct complete systems of solutions. This is carried out in $\S \S 2-4$. In $\S \S 5$ and 6 we discuss some related topics, while in the final sections we take up the problem of systems of equations. The work is applicable to linear differential equations, both ordinary and partial, in an arbitrary number of variables, or of systems of such equations.

2. Reduction to an interpolatory problem. For the sake of definiteness, but realizing that restrictions other than the ones about to be set forth may prove useful in other circumstances, we shall deal with $n$ complex variables

$$
z_{j}=x_{j}+i y_{j} \quad(j=1, \cdots, n),
$$

and shall designate by $B$ a fixed $2 n$-dimensional region in the space $Z=\left(z_{1}, \cdots\right.$, $z_{n}$ ) of the $n$ complex variables. We shall designate by $L^{2}(B)$ the class of functions $f$ which are single-valued analytic functions of $z$, are regular in $B$, and are such that

$$
\|f\|^{2}=\int_{B}|f|^{2} d \omega<\propto ; d \omega=d x_{1} \cdots d x_{n} d y_{1} \cdots d y_{n} .
$$

It may sometimes prove expedient to introduce a weight function in (2). By $L$, we shall designate a fixed linear operator defined on $L^{2}(B)$ and with the property that $L(f), f \in L^{2}(B)$, is regular analytic in $B$. Additional conditions on $L$ will be required below. We shall be concerned with representations of solutions of class $L^{2}(B)$ of the functional equation

$$
L(f)=0
$$


A principal application will be the case in which $L$ is a partial differential operator of the $k$ th order:

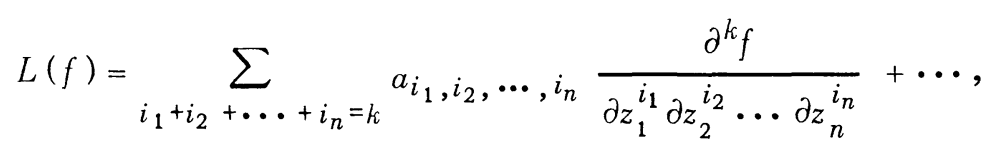

where the $+\cdots$ in (4) indicates the presence of partial derivatives of order $<k$. If now all the coefficients in (4) are regular in $B$, then so also will $L(f)$ be regular in $B$. It is to be remarked that the case $n=1$ which leads in (4) to an ordinary differential equation is not excluded.

Let $\left\{L_{n}\right\}(n=0,1 \ldots)$ be a sequence of linear functionals each of which is defined over the set $R$ of functions which are regular in $B$ and which possess the following two additional properties:

(a) The set $\left\{L_{n}\right\}$ is complete ${ }^{1}$ for $R$; that is, if $f \in R$ and $L_{n}(f)=0(n=0$, $1, \cdots)$, then $f \equiv 0$.

(b) Each linear functional $\widetilde{L}_{n} \equiv L_{n}(L)$ is bounded over $L^{2}(B)$; that is, for each $k$, there exists a positive constant $M_{k}$ such that

$$
\left|\widetilde{L}_{k}(f)\right|<M_{k}\|f\|
$$

for all $f \in L^{2}(B)$.

In connection with ( $b$ ), let us observe that the composite operator

$$
\widetilde{L}_{n}(f) \equiv L_{n}(L(f))
$$

is a linear functional from $L^{2}(B)$ onto the complex numbers.

Example. Let $L$ be the differential operator (4) with coefficients regular in $B$. Set

$$
L_{k}(f)=\left.\frac{\partial^{m_{1}+m_{2}+\cdots+m_{n}} f}{\partial z_{1}^{m_{1}} \partial z_{2}^{m_{2}} \cdots \partial z_{n}^{m_{n}}}\right|_{z_{j}=z_{j}^{*}}
$$

where $k=k\left(m_{1}, m_{2}, \cdots, m_{n}\right)$ refers to a fixed indexing of the $n$-tuples of nonnegative integers $m_{1}, m_{2}, \cdots, m_{n}$, and where the point $Z^{*}=\left(z_{1}^{*}, z_{2}^{*}, \ldots, z_{n}^{*}\right)$ is interior to $B$. It is clear that condition (a) holds for the selection (6). Let us

\footnotetext{
${ }^{1}$ Banach [2, p. 42] calls such sets total.
} 
next examine $\widetilde{L}_{n}$, which is (6) acting on (4). In this case $\widetilde{L}_{n}(f)$ is a finite linear combination with constant coefficients of mixed partial derivatives of $f$ evaluated at $Z=Z^{*}$. To show that condition (b) is satisfied, it suffices to show that any linear functional of the form (6), with $Z^{*}$ interior to $B$, is bounded over $L^{2}(B)$. This is a consequence of the fact that the functionals $(6)$ have a representation as Cauchy integrals with the path of integration lying in $B$, and hence are applicable term by term to any series of analytic functions which converges uniformly in a neighborhood of $Z^{*}$. Now let

$$
\phi_{m}(Z)=\phi_{m}\left(z_{1}, z_{2}, \cdots, z_{n}\right)
$$

be a complete orthonormal system for $L^{2}(B)$. Each $f \in L^{2}(B)$ possesses a Fourier expansion

$$
f(Z)=\sum_{n=0}^{\infty} a_{n} \phi_{n}(Z) ; \sum_{n=0}^{\infty}\left|a_{n}\right|^{2}=\|f\|^{2}<\propto,
$$

convergent uniformly in every closed bounded subregion of $B$. Hence

$$
L_{k}(f)=\sum_{n=0}^{\infty} a_{n} L_{k}\left(\phi_{n}(Z)\right),
$$

the series (8) converging for all selections $a_{n}$ with $\sum_{n=0}^{\infty}\left|a_{n}\right|^{2}<\infty$. By a lemma of Landau, this implies that

$$
\sum_{n=0}^{\infty}\left|L_{k}\left(\phi_{n}(Z)\right)\right|^{2}<\infty
$$

and the Schwarz inequality applied to (8) yields

$$
\left|L_{k}(f)\right|^{2} \leq\|f\|^{2} \sum_{n=0}^{\infty}\left|L_{k}\left(\phi_{n}\right)\right|^{2}
$$

This establishes ( $b$ ).

For analytic functions of a single complex variable, complete sets of functionals $\left\{L_{n}\right\}$ of a wide variety are known. We can, for instance, replace (6) (in the 1-dimensional case) by

$$
L_{k}(f)=f\left(Z_{k}\right) ; \lim _{k \rightarrow \infty} Z_{k}=Z^{*} ; Z_{k}, Z^{*} \text { interior to } B
$$


If we are dealing with differential equations with nonconstant coefficients, such a selection may reduce the complexity of the subsequent formal work. The points $Z_{k}$ need not have an accumulation point interior to $B$ as is suggested by (11), but, as in the Blaschke theory for the unit circle, may only have a weak accumulation of points on the boundary. In the theory of analytic functions of several complex variables, questions of the completeness of linear functionals are largely uninvestigated. However, certain sets in addition to (6) are known. Thus, for example, we may select the set (11) with the added restriction that the points $Z_{k}$ do not lie on an analytic hypersurface [10, p.39].

The functionals (6) and (11) are the usual "point" functionals met in interpolatory function theory. However, complete sets of integral functionals usually associated with orthogonal expansions may also be employed here. Within an $L^{2}$ theory, for complex analytic functions the distinction between these two types is weak, and persists only in certain discussions [8].

Under the foregoing hypotheses, we have the following result.

THEOREM 1. The linear functional equation $L(u)=0$ possesses a nontrivial solution of class $L^{2}(B)$ if and only if the set of functionals $\left\{\widetilde{L}_{n}\right\}$ is incomplete for $L^{2}(B)$.

Proof. Suppose first that $\left\{\widetilde{L}_{k}\right\}$ is incomplete. Then there exists an $f \in L^{2}(B)$ which does not vanish identically and such that $\widetilde{L}_{k}(f)=0 \quad(k=0,1, \cdots)$. That is, $L_{k}(L(f))=0$ for $k=0,1, \ldots$. By hypothesis, $L(f)$ is regular in $B$. Since therefore $\left\{L_{k}\right\}$ is complete for the class of regular functions in $B$, we must have $L(f) \equiv 0$. Conversely, let these begin a nontrivial $f \in L^{2}(B)$ such that $L(f) \equiv 0$ in $B$. Then

$$
\widetilde{L}_{k}(f)=L_{k}(L(f))=0 \text {, }
$$

so that the incompleteness of $\left\{\widetilde{L}_{k}\right\}$ follows.

In this way, the consideration of the functional equation $L(f)=0$ may be reduced to a consideration of the denumerable infinity of interpolation conditions of the type ${ }^{2}$

$$
\widetilde{L}_{k}(f)=0
$$$$
(k=0,1, \cdots) \text {. }
$$

It is frequently of importance to be able to solve this equation subject to the auxiliary conditions

\footnotetext{
${ }^{2}$ Interpolation problems of this type, where the functionals involved are point functionals have been considered in Bergman, Mémorial Sciences Math., vol. 106, pp. 46-48.
} 


$$
A_{n}(f)=0
$$$$
(n=0,1,2, \cdots) \text {, }
$$

where $A_{n}$ designates a linear functional which we shall again assume is bounded over $L^{2}(B)$. Let now $\left\{\hat{L}_{n}\right\}$ be an augmented set of linear functionals which includes both the sets $\left\{\widetilde{L}_{n}\right\}$ and $\left\{A_{n}\right\}$, but only these; that is, each $\hat{L}_{n}$ is either an $\widetilde{L}_{n}$ or an $A_{n}$, while every $\widetilde{L}_{n}$ and every $A_{n}$ is some $\hat{L}_{n}$. We may now state the following result.

THEOREM 1'. The linear functional equation (3), under the auxiliary conditions (12), possesses a nontrivial solution of class $L^{2}(B)$ if and only if the set of linear functionals $\left\{\hat{L}_{n}\right\}$ is incomplete for $L^{2}(B)$.

Thus it appears that, from our present point of view; the role played by the auxiliary conditions (12) is indistinguishable from that of the functional equation itself. In the notation used later, the circumflex $\wedge$ will indicate the presence of auxiliary conditions; that is, we deal with the equation (3) and derive from it a set of functionals $\left\{\widetilde{L}_{n}\right\}$, but when auxiliary conditions are present, the set $\left\{\widetilde{L}_{n}\right\}$ will be augmented to yield $\left\{\hat{L}_{n}\right\}$. It should also be observed that, in eigenvalue problems, the operator $L$ may involve a parameter $\lambda$. In such cases, the functionals $\widetilde{L}_{n}$ and $\hat{L}_{n}$ will also involve this parameter.

3. Representation of solutions. We reproduce here, for convenience of reference, the following theorem on double orthogonality which was established in a previous paper [12].

THEOREM 2. Let $\left\{\widetilde{L}_{k}\right\}$ be a set of linear functionals each of which is defined and bounded over $L^{2}(B)$. The set $\left\{\widetilde{L}_{k}\right\}$ will be assumed independent. There then exists a set of functions $\left\{\phi_{k}^{*}(Z)\right\}(k=0,1, \cdots)$ and a set of linear functionals $\left\{\widetilde{L}_{k}^{*}\right\}(k=0,1, \cdots)$ which possess the following properties:

(a) Each $\phi_{k}^{*}$ is of class $L^{2}(B)$ and the set is orthonormal over B:

$$
\int_{B} \phi_{i}^{*} \overline{\phi_{k}^{*}} d \omega=\delta_{i k} \text {. }
$$

(b) Each $\widetilde{L}_{k}^{*}$ is a finite linear combination of the functionals $\widetilde{L}_{k}$ :

$$
\widetilde{L}_{k}^{*}=\sum_{p=0}^{k} a_{k p} \widetilde{L}_{p}
$$$$
(k=0,1, \cdots)
$$ 
(c) The sets $\left\{\widetilde{L}_{k}^{*}\right\}$ and $\left\{\phi_{k}^{*}\right\}$ are biorthonormal:

$$
\widetilde{L}_{i}^{*}\left(\phi_{k}^{*}\right)=\delta_{i k} \cdot
$$

(d) For all $f \in L^{2}(B)$, we have

$$
\widetilde{L}_{k}^{*}(f)=\int_{B} f \overline{\phi_{k}^{*}} d \omega .
$$

(e) The functions $\phi_{k}^{*}$ may be obtained by taking the set ${ }^{2}$

$$
\phi_{n}(Z)=\widetilde{L}_{n, \bar{w}} K_{B}(Z, \bar{W}) \quad(n=0,1, \cdots)
$$

and orthonormalizing them by the Gram-Schmidt process.

(f) The set $\left\{\phi_{k}^{*}\right\}$ is complete for $L^{2}(B)$ if and only if the set $\left\{\widetilde{L}_{k}\right\}$ is complete for $L^{2}(B)$.

In (17),

$$
K_{B}(Z ; \bar{W})=K_{B}\left(z_{1}, z_{2}, \cdots, z_{n} ; \bar{w}_{1}, \bar{w}_{2}, \cdots, \bar{w}_{n}\right)
$$

designates the Bergman kernel function for the domain $B$, and in our notation an asterisk * used with the symbols for either functions or functionals indicates that the corresponding set of functions or functionals is orthonormal. Starting from a given $B$ and a given ordered set $\left\{\widetilde{L}_{k}\right\}$, the sets $\left\{\widetilde{L}_{k}^{*}\right\}$ and $\left\{\phi_{k}^{*}\right\}$ are determined uniquely, and we shall speak of them as being the biorthogonal sets associated with $\left\{\widetilde{L}_{k}\right\}$ and $B$.

The inner products

$$
\left(\phi_{i}, \phi_{j}\right)=\int_{B} \phi_{i} \overline{\phi_{j}} d \omega
$$

which occur in the orthonormalizing process, may be easily evaluated in terms of $K_{B}(Z ; \bar{W})$. We have, from $(16),(17)$, and the orthonormal expansion for $K_{B}$,

$$
\left(\phi_{i}, \phi_{j}\right)=L_{j, z}\left[L_{i, \bar{w}} K_{B}(Z ; \bar{W})\right] .
$$

If we introduce the determinants

$$
D_{n}=\left|\left(\phi_{i}, \phi_{j}\right)\right| \quad(i, j=0,1, \cdots, n),
$$

\footnotetext{
${ }^{3}$ The notation $L_{n}, \bar{w}$ means that $L_{n}$ is to be applied to $K_{B}$ as a function of $\bar{w}$.
} 
then we have

(21)

$$
\phi_{n}^{*}(Z)=\left(D_{n-1} D_{n}\right)^{-1 / 2} \cdot\left|\begin{array}{ccc}
\left(\phi_{0}, \phi_{0}\right), & \cdots, & \left(\phi_{0}, \phi_{n}\right) \\
\cdot & \\
\cdot & \\
\left(\phi_{n-1}, \phi_{0}\right), \ldots, & \left(\phi_{n-1}, \phi_{n}\right) \\
\phi_{0}(Z), & \cdots, & \phi_{n}(Z)
\end{array}\right|
$$

while

$$
\begin{aligned}
& a_{n i}=(-1)^{i}\left(D_{n-1} D_{n}\right)^{-1 / 2} \\
& \left|\begin{array}{cccc}
\left(\phi_{0}, \phi_{0}\right), \cdots,\left(\phi_{0}, \phi_{i-1}\right), & \left(\phi_{0}, \phi_{i+1}\right), & \cdots, & \left(\phi_{0}, \phi_{n}\right) \\
\cdot & \cdot & \cdot & \cdot \\
\cdot & \cdot & \cdot & \cdot \\
\left(\phi_{n-1}, \phi_{0}\right), \ldots,\left(\phi_{n-1}, \phi_{i-1}\right),\left(\phi_{n-1}, \phi_{i+1}\right), \ldots,\left(\phi_{n-1}, \phi_{n}\right)
\end{array}\right| .
\end{aligned}
$$

In view of the orthonormality of the functions $\phi_{k}^{*}$, we may form the kernel function

$$
K_{I}(Z ; \bar{W})=\sum_{k=0}^{\infty} \phi_{k}^{*}(Z) \overline{\phi_{k}^{*}(W)},
$$

the series (23) converging uniformly and absolutely for $Z$ and $W$ confined to any closed bounded subset of $B \times B$ and defining there an analytic function of $z_{1}, z_{2}, \cdots, z_{n}$ and an anti-analytic function of $w_{1}, w_{2}, \cdots, w_{n}$. If the system $\left\{\phi_{k}^{*}\right\}$ is complete for $L^{2}(B)$, then $K_{I}$ must coincide with $K_{B}$. If auxiliary conditions are present, we replace $(23)$ by

$$
K_{I}(Z ; \bar{W})=\sum_{k=0}^{\infty} \hat{\phi}_{k}^{*}(Z) \overline{\left[\hat{\phi}_{k}^{*}(\mathbb{W})\right]},
$$

where $\left\{\hat{\phi}_{k}^{*}\right\}$ and $\left\{\hat{L}_{k}^{*}\right\}$ are the biorthonormal sets associated with $\left\{\hat{L}_{k}^{*}\right\}$ and $B$.

Combining this observation with Theorem 1 , we have the following result:

THEOREM 2. The functional equation (3) (augmented, possibly, by 
auxiliary conditions (12)) possesses nontrivial solutions of class $L^{2}(B)$ if and only if $K_{I} \equiv K_{B}$, or if and only if $K_{I}(Z ; \bar{Z})<K_{B}(Z ; \bar{Z}), Z \in B$.

If we admit the possibility of a nontrivial solution, the kernel $K_{I}$ may be thought of as an "incomplete" kernel for $B$ relative to the functional equation (3). It is wholly accessible to computation via (19)-(23) once $K_{B}$ has been established. Moreover, $K_{I}$ may be used to project the space $L^{2}(B)$ onto the linear subspace $S$ of solutions:

THEOREM 3. The function $g(Z)$ is a solution of (3) of class $L^{2}(B)$ if and only if there exists an $f \in L^{2}(B)$ for which

$$
T(f) \equiv g(Z)=f(Z)-\int_{B} K_{I}(Z, \bar{W}) f(\mathbb{W}) d \omega_{\mathbb{W}},
$$

or alternately, for which

$$
T(f) \equiv g(Z)=f(Z)-\sum_{k=0}^{\infty} \widetilde{L}_{k}^{*}(f) \phi_{k}^{*}(Z)
$$

Appropriate changes must be made if auxiliary conditions (12) are present.

Proof. We observe that in view of (16) and (23), (24) and (24,) are equivalent. For a given $f \in L^{2}(B)$, construct a $g$ by means of $\left(24^{\prime}\right)$. Since the quantities $\widetilde{L}_{n}^{*}(f)$ are Fourier coefficients of $f$, the sum in $\left(24^{\prime}\right)$ is of class $L^{2}(B)$. Thus also $g \in L^{2}(B)$. As remarked previously, $g$ will be a solution of $(3)$ if $\widetilde{L}_{k}(g)=0 \quad(k=0,1, \cdots)$. By $(14)$, this is equivalent to $\widetilde{L}_{k}^{*}(g)=0 \quad(k=$ $0,1, \cdots)$. In view of the boundedness of $\widetilde{L}_{k}^{*}$ over $L^{2}(B)$, we have

$$
\widetilde{L}_{k}^{*}(g)=\widetilde{L}_{k}^{*}(f)-\sum_{n=0}^{\infty} \widetilde{L}_{n}^{*}(f) \widetilde{L}_{k}^{*}\left(\phi_{n}^{*}\right)=\widetilde{L}_{k}^{*}(f)-\widetilde{L}_{k}^{*}(f)=0 \text {. }
$$

The last equality follows from ( 15$)$. Thus $g$ is a solution. Conversely, if $g$ is a solution of class $L^{2}(B)$ we shall have

$$
\widetilde{L}_{k}^{*}(g)=L_{k}^{*}(L(g))=0 \quad(k=0,1, \cdots),
$$

so that $\left(24^{\prime}\right)$ holds with $f \equiv g$.

Equation (24)-(24,) yields a projection of $L^{2}(B)$ onto the subspace $S$ of solutions. The partial sums of $\left(24^{\prime}\right)$, 


$$
\sum_{k=0}^{N} \widetilde{L}_{k}^{*}(f) \phi_{k}^{*}(Z),
$$

have the usual minimum property of Fourier series; that is, for each $N$ they solve the minimization of the integral

$$
I_{N}=\int_{B}\left|f-\sum_{k=0}^{N} a_{k} \phi_{k}(Z)\right|^{2} d \omega .
$$

On the other hand, the series in (24') has two characters: it is simultaneously a Fourier series and an interpolation series as well. This means that the partial sum

$$
S_{N}=\sum_{k=0}^{N} \widetilde{L}_{k}^{*}(f) \phi_{k}^{*}(Z)
$$

is that linear combination of $\phi_{0}, \phi_{1}, \cdots, \phi_{N}$ which interpolates to $f$ in the sense that

$$
\widetilde{L}_{k}^{*}\left(S_{N}(Z)\right)=\widetilde{L}_{k}^{*}(f) \quad(k=0,1, \cdots, N),
$$

or, equivalently,

$$
\widetilde{L}_{k}\left(S_{N}(z)\right)=\widetilde{L}_{k}(f)
$$$$
(k=0,1, \cdots, N) \text {, }
$$

Cnce $K_{B}$ is known, and if $\left\{L_{k}\right\}$ is a sequence of point or differential operators, then no integrals extended over $B$ of the inner product type need actually be computed to obtain either $\phi_{n}^{*}$ or the series expansion in $\left(24^{\prime}\right)$. The explicit orthogonalization formulas of Gram-Schmidt (20)-(22) are equivalent to an interpolation series of Newton type with respect to the sequence $\left\{\widetilde{L}_{n}\right\}$. For a fixed $N$, formulas of the Lagrange type may be developed, and may prove to be more convenient.

In view of the reproducing property of $K_{B}$, we may write $(24)$ in the form

$$
T(f)=g(Z)=\int_{B} K_{B}(Z ; \bar{W}) f(\mathbb{W}) d \omega_{W}-\int_{B} K_{I}(Z ; \bar{W}) f(\mathbb{W}) d \omega_{W},
$$

so that by introducing the kernel 


$$
K_{S}(Z ; \bar{W})=K_{B}(Z ; \bar{W})-K_{I}(Z ; \bar{W})
$$

we have the representation

$$
g(Z)=\int_{B} K_{S}(Z ; \bar{W}) f(\mathbb{W}) d \omega_{W}
$$

If $f \in S$, then $g(Z) \equiv f(Z)$ inasmuch as

$$
\widetilde{L}_{n}^{*}(f)=L_{n}^{*}(L(f))=0 \quad(n=0,1, \cdots) .
$$

Thus, $K_{S}(Z ; \bar{W})$ is a reproducing kernel for the subspace $S$ and as such, may be proved unique (that is, nondependent upon the selection $\left\{L_{n}\right\}$ ) in the usual way. $K_{S}(Z, \bar{W})$ may also be defined by

$$
K_{S}(Z ; \bar{W})=\sum_{k} \psi_{k}(Z) \overline{\psi_{k}(\mathbb{W})}
$$

where $\left\{\psi_{k}\right\}$ is any orthonormal set which is complete for $S$. In the case of ordinary differential equations, the sum in (32) will consist of a finite number of terms. In the case of ordinary differential equations of infinite order or of partial differential equations, there will, in general, be an infinity of terms present.

The incomplete kernel $K_{I}$ may be identified as the kernel of the orthogonal complement $S^{\perp}$ of $S$, and the utility of the backward decomposition of $K_{B}$ given by (30) lies in relative accessibility of $K_{I}$ as opposed to $K_{S}$. Let us note also the orthogonality relationship

$$
\int_{B} K_{S}(Z ; \bar{W}) K_{I}(W ; \bar{X}) d \omega_{W}=0,
$$

which follows from (30) and from the reproducing properties of $K_{S}$ and $K_{B}$ over $S$ and $L^{2}(B)$, respectively.

For $f \in S$, we have, by (31) and the Schwarz inequality,

$$
\begin{aligned}
|f|^{2}<\|f\|^{2} \int_{B} K_{S}(Z ; \bar{W}) K_{S}(\bar{Z} ; \mathbb{W}) & d \omega_{W} \\
& =\|f\|^{2}\left[K_{B}(Z ; \bar{Z})-K_{I}(Z ; \bar{Z})\right] .
\end{aligned}
$$

The inequality (34) is a wide generalization of the Schwarz Lemma for functions 
regular in the unit circle. Finally, we may generate complete sets of solutions in the following way:

THEOREM 4. Let $\theta_{n}(Z) \quad(n=0,1, \cdots)$ be a complete set for $L^{2}(B)$; then the functions

$$
\begin{aligned}
\psi_{n}(Z)=T\left(\theta_{n}\right) & =\int_{B} K_{S}(Z ; \overline{\mathbb{W}}) \theta_{n}(\mathbb{W}) d \omega_{\mathbb{W}} \\
& =\theta_{n}(Z)-\sum_{k=0}^{\infty} \widetilde{L}_{k}^{*}\left(\theta_{n}\right) \phi_{k}^{*}(Z) \quad(n=0,1, \cdots)
\end{aligned}
$$

form a complete set of solutions.

Proof. We must show that any solution $g$ can be approximated arbitrarily closely by combinations of $\psi_{0}, \cdots, \psi_{n}, \cdots$. Let

$$
\left\|g-\sum_{k=0}^{N} a_{k} \theta_{k}\right\|<\epsilon .
$$

Then by (34), (35), we have

$$
\left|g-\sum_{k=0}^{N} a_{k} \psi_{k}\right|<\epsilon\left[K_{B}(Z ; \bar{Z})-K_{I}(Z ; \bar{Z})\right]^{1 / 2},
$$

which establishes completeness.

4. The nonhomogeneous case. We consider next the nonhomogeneous linear functional equation

$$
L(u)=f \text {, }
$$

which may be supplemented by auxiliary conditions of the form

$$
A_{k}(u)=\alpha_{k} \quad(k=0,1, \cdots) .
$$

We assume that $f$ is regular in $B$, and that all previous hypotheses regarding $L$ and $A_{n}$ remain in force. We first reduce (36)-(37) to a problem in interpolation in the following way.

THEOREM 5. The linear functional equation (36), subject to the auxiliary 
conditions (37), is equivalent to the interpolation problem

$$
\widetilde{L}_{k}(u)=L_{k}(f)
$$

$$
A_{k}(u)=\alpha_{k}
$$$$
(k=0,1, \cdots) \text {. }
$$

If conditions (37) are absent we may omit (38').

Proof. That (38), (38') follow from (36), (37) is evident. Suppose conversely that $(38)$ holds. We wish to prove that $L(u) \equiv f$ throughout $B$. We have

$$
L_{k} L(u)-L_{k}(f)=0 \quad(k=0,1, \cdots) \text {. }
$$

Thus

$$
L_{k}[L(u)-f]=0 \quad(k=0,1, \cdots) .
$$

Now $L(u)-f$ is regular in $B$, and $\left\{L_{k}\right\}$ is complete for $R$. Hence the conclusion follows.

It will now be convenient to uniformize our notation. We introduce an augmented set $\left\{\hat{L}_{k}\right\}$ of linear functionals as in the previous paragraph, and introduce a set of constants $\left\{\beta_{k}\right\}$ by means of the definition

$$
\begin{array}{ll}
\beta_{k}=L_{k}(f) & \text { if } \hat{L}_{k}=\widetilde{L}_{k}, \\
\beta_{k}=\alpha_{k} & \text { if } \hat{L}_{k}=A_{k} .
\end{array}
$$

The interpolation problem is now

$$
\hat{L}_{k}(u)=\beta_{k}
$$$$
(k=0,1, \cdots) \text {. }
$$

We observe again that there is no distinct rôle played by the auxiliary conditions. Boundary value and initial value problems of mathematical physics may be fitted into the pattern (40) providing it is known a priori that the required solutions are regular across the boundary so that the functionals $\hat{L}_{k}$ will have the required boundedness properties. We next introduce the biorthonormal sets $\left\{\hat{L}_{k}^{*}\right\}$ and $\left\{\hat{\phi}_{k}^{*}\right\}$ associated with $\left\{\hat{L}_{k}\right\}$ and $B$. We have

$$
\hat{L}_{k}^{*}=\sum_{p=0}^{k} a_{k p} \hat{L}_{p}
$$

for constants $a_{k p}$ determined as in the previous paragraph. The following result 
now holds.

THEOREM 6. The linear functional equation (36), (37) possesses a solution of class $L^{2}(B)$ if and only if

$$
\sum_{k=0}^{\infty}\left|\sum_{p=0}^{k} a_{k p} \beta_{p}\right|^{2}<\infty .
$$

The solution is unique (within $L^{2}(B)$ ) if and only if $\left\{\hat{L}_{k}\right\}$ is complete for $L^{2}(B)$. If (42) holds, then the series

$$
u(Z)=\sum_{k=0}^{\infty}\left(\sum_{p=0}^{k} a_{k p} \beta_{p}\right) \hat{\phi}_{k}^{*}(Z)
$$

converges to a solution $u(Z)$ uniformly and absolutely in every closed bounded subset of $B$.

Proof. Suppose that a solution $u \in L^{2}(B)$ exists. Then from (40) and (4.1) we have

$$
\hat{L}_{k}^{*}(u)=\sum_{p=0}^{k} a_{k p} \beta_{p} .
$$

But since the $\hat{L}_{k}^{*}(u)$ are Fourier coefficients of $u$ with respect to $\left\{\phi_{k}^{*}\right\}$, we must have (42). If ( 42$)$ holds, then the series (43) converges uniformly and absolutely in every closed bounded subregion of $B$ to a function $V(Z)$ of class $L^{2}(B)$. Now,

$$
\hat{L}_{k}^{*}(V)=\sum_{p=0}^{k} a_{k p} \beta_{p}
$$

in view of the boundedness and biorthogonality properties of these functionals. Hence

$$
\hat{L}_{k}(V)=\beta_{k} \quad(k=0,1, \cdots) .
$$

so that $V$ satisfies the equations (36) and (37) by Theorem 5 .

A particularly important special case is to solve (36) subject to the auxiliary 
conditions

$$
A_{k}(u)=0
$$$$
(k=0,1, \cdots) .
$$

As in Theorem 5, we again construct the biorthonormal sets $\left\{\hat{L}_{k}^{*}\right\}$ and $\left\{\hat{\phi}_{k}^{*}\right\}$, and note that each functional $\hat{L}_{k}^{*}$ is a finite linear combination of functionals $\widetilde{L}_{k}$ and $A_{k}$ :

$$
\hat{L}_{k}^{*}=\sum_{p=0}^{k} a_{k p} \hat{L}_{p}=\sum_{p} b_{k p} \widetilde{L}_{p}+\sum_{p} c_{k p} A_{p},
$$

where the coefficients $b_{k p}$ and $c_{k p}$ now contain certain dummy zeros. Let us write

$$
\sum_{p} \dot{b}_{k p} \widetilde{L}_{p}=\sum_{p} b_{k p} L_{p} L=S_{k} L ; S_{k}=\sum_{p} b_{k p} L_{p}
$$

We now have the following result.

THEOREM 6'. The linear functional equation (36), (44) possesses a solution of class $L^{2}(B)$ if and only if

$$
\sum_{k=0}^{\infty}\left|S_{k}(f)\right|^{2}<\infty
$$

If (47) holds, the interpolation series

$$
u(Z)=\sum_{k=0}^{\infty} S_{k}(f) \hat{\phi}_{k}^{*}(Z)
$$

converges to a solution uniformly and absolutely in every closed bounded subset of $B$.

Proof. If $\beta_{k}=\alpha_{k}=0$ when $\hat{L}_{k}=A_{k}$, then, by (39a),

$$
\sum_{p} a_{k p} \beta_{p}=\sum_{p} b_{k p} L_{p}(f)=S_{k}(f) .
$$

Under the assumption that the equation (36), (44) possesses a solution for 
all $f \in L^{2}(B)$, we may find a second representation for the interpolation series (48). The functionals $S_{k}$ are bounded over $L^{2}(B)$, and hence possess a Riesz representative $s_{k}(Z)$ :

$$
S_{k}(f)=\int_{B} f \bar{s}_{k} d \omega ; f \in L^{2}(B),
$$

where

$$
s_{k}(Z)=S_{k, \bar{w}} K_{B}(Z ; \bar{W}) .
$$

If (36), (44) possess a solution of class $L^{2}(B)$ for all $f \in L^{2}(B)$, then (47) must hold for all $f \in L^{2}(B)$. In particular, from

$$
\overline{s_{k}\left(Z_{0}\right)}=S_{k, w} K_{B}\left(\bar{Z}_{0} ; W\right), Z_{0} \in B,
$$

we learn that

$$
\sum_{k=0}^{\infty}\left|s_{k}\left(Z_{0}\right)\right|^{2}<\infty
$$

We may therefore form the mixed kernel

$$
D(Z ; \bar{W})=\sum_{k=0}^{\infty} \hat{\phi}_{k}^{*}(Z) \overline{s_{k}(\mathbb{W})},
$$

which will converge uniformly and absolutely in every closed bounded subregion of $B \times B$. Finally, from (48) and (49), we have the representation

$$
U(Z)=\int_{B} D(Z ; \bar{W}) f(\mathbb{W}) d \omega_{\mathbb{W}}
$$

The kernel $D(Z ; \bar{W})$ plays a role analogous to a Green's function or to the Duhamel kernel in the superposition theorem of the theory or ordinary linear differential equations. The totality of solutions in $L^{2}(B)$ of (36), (44) may be written in the form

$$
U(Z)=\int_{B} D(Z ; \bar{W}) f(\mathbb{W}) d \omega_{\mathbb{W}}+\int_{B} K_{s}(Z ; \bar{W}) h(W) d \omega_{W} ; h \in L^{2}(B),
$$

or, in interpolatory form, 


$$
U(Z)=\sum_{k=0}^{\infty} S_{k}(f) \hat{\phi}_{k}^{*}(Z)+h(Z)-\sum_{k=0}^{\infty} \hat{L}_{k}^{*}(h) \hat{\phi}_{k}^{*}(Z) ; h \in L^{2}(B) .
$$

5. Convergence of interpolation series for $f \notin L^{2}(B)$. In the present paragraph we return to the interpolation series $\left(24^{\prime}\right)$. This has been discussed under the hypothesis that $f \in L^{2}(B)$. If, however, each functional $\widetilde{L}_{k}^{*}$ ( or $\widetilde{L}_{k}$ ) is applicable to a wider class of functions than $L^{2}(B)$, a formal series (24') may be constructed and its properties examined for $f$ in this wider class. This will be the case, for example, when $L_{k}$ are differential operators. For the sake of definiteness, let us assume that we are dealing with the ordinary linear differential equation

$$
L(f) \equiv f^{(n)}+a_{1}(z) f^{(n-1)}+\cdots+a_{n}(z) f=0,
$$

and that we have selected

$$
L_{k}(f)=f^{(k)}(0) \quad(k=0,1, \cdots) .
$$

The coefficients $a_{j}(z)$ in (57) are assumed regular in a region $R$ containing the origin. If $f$ is regular at $z=0$, then the series $\left(24^{\prime}\right)$ may be formed. If this series then converges uniformly in a neighborhood of $z=0$, the difference

$$
g(z)=f(z)-\sum_{k=0}^{\infty} \widetilde{L}_{k}^{*}(f) \phi_{k}^{*}(z)
$$

which is again regular at $z=0$, will be a solution; for, since the functionals $\widetilde{L}_{k}^{*}$ are applicable term by term, we have

$$
\widetilde{L}_{k}^{*}(g)=\widetilde{L}_{k}^{*}(f)-\sum_{p=0}^{\infty} \widetilde{L}_{p}^{*}(f) \widetilde{L}_{k}^{*}\left(\phi_{p}^{*}\right)=\widetilde{L}_{k}^{*}(f)-\widetilde{L}_{k}^{*}(f)=0, \quad(k=0,1, \cdots)
$$

and this implies that $L(g)=0$. The interpolation series (24') has a doubly orthogonal character, but the above proof will apply to any interpolation series

$$
g(z)=f(z)-\sum_{k=0}^{\infty} \widetilde{L}_{k}^{*}(f) \psi_{k}(z)
$$

in which the regular functions $\psi_{k}$ are merely biorthogonal: 


$$
\widetilde{L}_{j}^{*}\left(\psi_{k}\right)=\delta_{j k}
$$

Such sets are more numerous than doubly orthogonal sets. To determine such a set, we need only start from a given set of functions $\left\{t_{n}(z)\right\} \quad(n=0,1, \ldots)$ which has properties of independence with respect to $\left\{\widetilde{L}_{j}\right\}$ and determine linear combinations

$$
\psi_{k}(z)=\sum_{p=0}^{k} e_{k p} t_{p}(z) \quad(k=0,1, \cdots)
$$

successively by the requirement (60).

We shall now prove that we may find a set $\left\{\psi_{k}(z)\right\}$ biorthogonal to $\left\{\widetilde{L}_{k}^{*}\right\}$ with the property that if $f$ is regular in any neighborhood of $z=0$, the interpolation series

$$
g(z)=f(z)-\sum_{k=0}^{\infty} \widetilde{L}_{k}^{*}(f) \psi_{k}(z)
$$

will converge to a solution of (57) in some neighborhood of $z=0$. The present proof will generalize to both partial differential equations and to ordinary differential equations of infinite order.

We have, from (57) and (58),

$$
\widetilde{L}_{k}(f)=\left.\sum_{p=0}^{n} \frac{d^{k}}{d z^{k}}\left[a_{p}(z) f^{(n-p)}(z)\right]\right|_{z=0}=\sum_{j=0}^{n+k} b_{k j} f^{(j)}(0),
$$

while

$$
\widetilde{L}_{k}^{*}(f)=\sum_{j=0}^{n+k} b_{k j}^{*} f^{(j)}(0)
$$

for appropriate $b_{j, k}^{*}$. We assume that $B$ contains the origin and is contained in the region of regularity of $a_{i}(z)$.

Lemma. Let $f(z)$ be regular in $|z| \leq \rho$. Then there exist positive constants $M$ and $t$ such that

$$
\left|\widetilde{L}_{k}^{*}(f)\right|<M t^{k} \quad(k=0,1, \cdots) .
$$


Proof. For any $g \in L^{2}(B)$, we have

$$
\widetilde{L}_{k}^{*}(g)=\iint_{B} g \bar{\phi}_{k}^{*} d x d y \quad(k=0,1, \cdots) .
$$

Thus $\widetilde{L}_{k}^{*}(g)$ are Fourier coefficients of $g$, so that, by the Bessel inequality,

$$
\sum_{k=0}^{\infty}\left|\widetilde{L}_{k}^{*}(g)\right|^{2}<\iint_{B}|g|^{2} d x d y .
$$

In particular, we may select

$$
g=z^{p} / p ! \quad(p=0,1, \cdots),
$$

so that

$$
\widetilde{L}_{k}^{*}\left(z^{p} / p !\right)=\sum_{j=0}^{n+k} b_{k j}^{*}\left[\left.z^{p / p !]^{(j)}}\right|_{z=0}=b_{k p}^{*} .\right.
$$

From (66) we obtain

$$
\begin{aligned}
\sum_{k=0}^{\infty}\left|\widetilde{L}_{k}^{*}\left(z^{p} / p !\right)\right|^{2}=\sum_{k=0}^{\infty}\left|b_{k p}^{*}\right|^{2} & \leq \iint_{B}\left|\frac{z^{p}}{p !}\right|^{2} d x d y \\
& \leq \frac{\operatorname{Area}(B)}{(p !)^{2}} d^{2 p}, \quad(p=0,1, \cdots),
\end{aligned}
$$

where $d$ designates the maximum distance from the boundary of $B$ to the origin. If now $f$ is regular in $|z| \leq \rho$, we have, for some constant $M^{*}$,

$$
\left|f^{(j)}(0)\right|<M^{*} j ! / \rho^{j} \quad(j=0,1, \cdots),
$$

so that from (64) and (68),

$$
\left|\widetilde{L}_{k}^{*}(f)\right|<M(d / \rho)^{k} \quad(k=0,1, \cdots),
$$

with

$$
M=M^{*}(\text { Area }(B))^{1 / 2}(d / \rho)^{n+1} /(d / \rho)-1 \text {. }
$$


LEMMA. There exist positive constants $m$ and $\sigma$ such that

$$
\left|L\left(\phi_{k}^{*}(z)\right)\right|<m|z|^{k}
$$

for all $k=0,1, \cdots$, and for all $|z| \leq \sigma$.

Proof. The orthonormal functions $\phi_{k}^{*}(z)$ satisfy the requirements $[12, \mathrm{p} .16]$

$$
\widetilde{L}_{0}\left(\phi_{k}^{*}\right)=\widetilde{L}_{1}\left(\phi_{k}^{*}\right)=\cdots=\widetilde{L}_{k-1}\left(\phi_{k}^{*}\right)=0 \text {, }
$$

or with notation $g_{k}=L \phi_{k}^{*}$,

$$
g_{k}(0)=g_{k}^{\prime}(0)=\cdots=g_{k}^{(k-1)}(0)=0 .
$$

Let $|z|=\sigma^{\prime}$ and $|z|=\sigma, \sigma^{\prime}>\sigma$ both be contained in $B$. Since $\phi_{n}^{*}$ are orthonormal over $B$, they are uniformly bounded by some $M$ over $|z| \leq \sigma^{\prime}$; hence, by (57) and Cauchy's i.ıequality,

$$
\left|L\left(\phi_{k}^{*}\right)\right| \leq \sigma^{\prime} M \sum_{j=0}^{n} B_{j} j ! s^{j+1}=m ;|z| \leq \sigma,
$$

where

$$
s=\sigma^{\prime}-\sigma \quad \text { and } \quad B_{j}=\max _{|z| \leq \sigma^{\prime}}\left|a_{j}(z)\right|
$$

Thus the functions $L\left(\phi_{k}^{*}\right)$ are uniformly bounded in $|z| \leq \sigma$ by $m$. The inequality (71) now follows from Schwarz's lemma.

We observe now that the last two lemmas imply that the series

$$
\sum_{k=0}^{\infty} \widetilde{L}_{k}^{*}(f) L \phi_{k}^{*}(z)
$$

will converge absolutely and uniformly in $|z| \leq r, r<1 / t$. Furthermore, we must have

$$
L(f)=\sum_{k=0}^{\infty} \widetilde{L}_{k}^{*}(f) L \phi_{k}^{*}(z) ;|z| \leq r .
$$

To show this, designate the sum of (73), $|z| \leq r$, by $g(z)$. By uniform 
convergence, we may apply $L_{p}$ term by term. Thus

$$
L_{p}(g)=\sum_{k=0}^{\infty} \widetilde{L}_{k}^{*}(f) L_{p} L \phi_{k}^{*}(z)
$$

so that

$$
L_{p}^{*}(g)=\sum_{k=0}^{\infty} \widetilde{L}_{k}^{*}(f) \widetilde{L}_{p}^{*}\left(\phi_{k}^{*}(z)\right)=\widetilde{L}_{p}^{*}(f)=L_{p}^{*} L(f)
$$

By the completeness of $\left\{L_{p}^{*}\right\}, g \equiv L(f)$.

Let now $B_{1}$ designate a region containing $z=0$ and contained in $|z|<r$, and let $D(z, \bar{w}) \equiv D_{B_{1}}(z, \bar{w})$ be the kernel described in (54)-(55). We have, for each $f$ regular in $|z| \leq r$, the identity

$$
f(z)=\iint_{B_{1}} D(z, \bar{w}) L(f(w)) d \omega_{w}+s(z),
$$

where $s(z)$ is some solution of $L(s)=0$, regular in $B_{1}$. Applying this inversion operator to $(73)$, we have

$$
\begin{aligned}
f(z)-s(z) & =\sum_{k=0}^{\infty} \widetilde{L}_{k}^{*}(f) \iint_{B_{1}} D(z, \bar{w}) L\left(\phi_{k}^{*}(w)\right) d \omega_{w} \\
& =\sum_{k=0}^{\infty} \widetilde{L}_{k}^{*}(f) \psi_{k}(z),
\end{aligned}
$$

where

$$
\psi_{k}(z)=\iint_{B_{1}} D(z, \bar{w}) L\left(\phi_{k}^{*}(w)\right) d \omega_{w} \quad(k=0,1, \cdots)
$$

The functions $\left\{\psi_{k}\right\}$ are easily seen to be biorthonormal to the interpolation operators $\left\{\widetilde{L}_{k}^{*}\right\}$. We therefore have the following result.

THEOREM 7. For each $f(z)$ regular in $|z| \leq \sigma$, the biorthogonal interpolation series 


$$
g(z)=f(z)-\sum_{k=0}^{\infty} \widetilde{L}_{k}^{*}(f) \psi_{k}(z)
$$

converges to a solution of the equation (57).

6. Relation to questions of stability. In a previous paragraph we have given necessary and sufficient conditions in order that a given functional equation possess solutions of class $L^{2}(B)$. If the coefficients of this equation involve

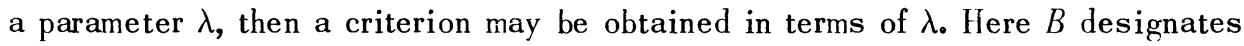
any region which possesses a kernel function $K_{B}$. If $B$ is chosen as an unbounded domain, then membership in $L^{2}(B)$ acts as a stability criterion.

To elucidate this remark, let us consider the two dimensional case, and let $S$ designate the half-strip

$$
\operatorname{Re}(z) \geq 0,|\operatorname{Im}(z)| \leq h .
$$

Then we have $f \in L^{2}(S)$ if and only if ${ }^{4}$

$$
\|f\|_{S}^{2}=\int_{-h}^{h} \int_{0}^{\infty}|f(x+i y)|^{2} d x d y<\infty .
$$

Thus, to belong to $L^{2}(S)$ a function must not become large too rapidly as $z$ approaches the horizontal boundaries of the strip, and indeed, must approach zero with a certain maximal rapidity along any horizontal line.

Lemma. Let $f \in L^{2}(S)$; then along each line

$$
y=\sigma,-h<\sigma<h,
$$

we must have

$$
\lim _{x \rightarrow+\infty} f(x+i \sigma)=0
$$

Proof. If (81) were not true, we could find two positive quantities $A$ and $\delta$ and a sequence of values $\lambda_{0}<\lambda_{1}<\ldots$ such that

$$
\lambda_{n}-\lambda_{n-1} \geq \delta>0 \quad(n=1,2, \ldots),
$$

and [13].

${ }^{4}$ Various authors have considered solutions of class $L^{2}(0, \infty)$; for example, see 
( 83 )

$$
\left|f\left(\lambda_{n}+i \sigma\right)\right| \geq A>0
$$$$
(n=0,1, \cdots) \text {. }
$$

In virtue of (82) we may find an $r>0$ such that the circles

$$
C_{n}:\left|z-\left(\lambda_{n}+i \sigma\right)\right| \leq r
$$

lie in $S$ and do not overlap. Now

$$
\propto>\iint_{S}|f|^{2} d x d y>\sum_{n=0}^{\infty} \iint_{C_{n}}|f|^{2} d x d y .
$$

Since $f$ is regular in $C_{n}$, it possesses a Taylor series expansion

$$
f(z)=f\left(P_{n}\right)+f^{\prime}\left(P_{n}\right)\left(z-P_{n}\right)+\cdots ; P_{n} \equiv \lambda_{n}+i \sigma,
$$

so that

$$
\iint_{C_{n}}|f(z)|^{2} d x d y>\pi r^{2}\left|f\left(P_{N}\right)\right|^{2} .
$$

Combining this with (84), we must have

$$
\sum_{n=0}^{\infty}\left|f\left(P_{n}\right)\right|^{2}<\infty
$$

This contradicts ( 83$)$ and proves the result.

The 'stability' which is spoken of here is that usually associated with the theory of linear, non time-varying electrical networks; in this theory we are confronted with a differential equation

$$
y^{(n)}+a_{1} y^{(n-1)}+\cdots+a_{n} y=f(x),
$$

to be solved under initial conditions such as

$$
y(0)=y^{\prime}(0)=\cdots y^{(n-1)}(0)=0 .
$$

If the characteristic roots of (88) are

$$
\tau_{j}=u_{j}+i v_{j}
$$$$
(j=1,2, \cdots, n),
$$

assumed distinct, then the $n$ independent solutions of the homogeneous equation 
are

$$
y_{i}(x)=e^{u_{j} x} e^{i v_{j} x}
$$

The equation ( 88$)$ is called stable if $u_{j}<0(j=1, \cdots, n)$. We observe now that $y_{j}(z) \in L^{2}(S)$ if and only if $u_{j}<0$. For

$$
\left|y_{j}(z)\right|^{2}=e^{2\left(u_{j} x-v_{j} y\right)}
$$

and this result is now implied by ( 80$)$. It appears then that the equation ( 88 ) is stable if and only if the fundamental solutions of the homogeneous equation are in $L^{2}(S)$. For the case of more general linear networks, we propose membership in $L^{2}(S)$ as a possible extension of this type of stability. Added flexibility may be achieved by varying $h$, and by attaching a weighting function to (80). Inasmuch as the mapping function for $S$ is elementary, the kernel function $K_{S}$ of $S$ may be computed explicitly, and the criterion of $\S 3$ can be formulated in closed form.

7. Systems of functional equations. The methods of the previous paragraphs may be extended to the case of systems of equations. As the proofs and the principal results parallel those given in $\$ 2-4$ very closely, we shall not dwell on these aspects, and shall be content merely with showing how the generalization may be set up.

For the sake of simplicity, we consider here only systems of two functional equations in the two unknown functions, $u_{i}=u_{i}\left(z_{1}, z_{2}, \ldots, z_{n}\right)=u_{i}(Z),(i=1,2)$,

$$
\begin{aligned}
& L^{1}\left(u_{1}, u_{2}\right)=0, \\
& L^{2}\left(u_{1}, u_{2}\right)=0 .
\end{aligned}
$$

Introducing the solution vector $\mathbf{u}=\left(u_{1}, u_{2}\right)$ and the vector operator $\mathbf{L}=\left(L_{1}, L_{2}\right)$, we may write $(90)$ as

$$
\mathbf{L}(\mathbf{u})=0 \text {. }
$$

We assume that $L^{i}\left(u_{1}, u_{2}\right)$ are regular functions of $z_{1}, \cdots, z_{n}$ whenever $u_{i}$ are, and that $\mathbf{L}$ is linear on the vector $\mathbf{u}$. We shall say that $\left(90^{\prime}\right)$ possesses a solution of class $L^{2}(B)$ if there exists $u_{i} \in L^{2}(B)$ for which $\left(90^{\prime}\right)$ holds. In addition to (90), we may consider an augmented system comprising (90) plus certain auxiliary conditions which may be written in the form 


$$
\mathbf{A}_{n}(\mathbf{u})=A_{n}\left(u_{1}, u_{2}\right)=0 \quad(n=0,1, \ldots) .
$$

Here $\mathbf{A}_{n}$ is a linear functional on u. Let again $\left\{L_{n}\right\}$ designate a fixed set of linear functionals defined on the set of functions regular on $B$ and complete for this set. We introduce

$$
\begin{array}{ll}
\tilde{\mathbf{L}}_{2 n}(\mathbf{u})=L_{n} L^{1}\left(u_{1}, u_{2}\right) & (n=0,1, \cdots), \\
\tilde{\mathbf{L}}_{2 n+1}(\mathbf{u})=L_{n} L^{2}\left(u_{1}, u_{2}\right) & (n=0,1, \cdots) .
\end{array}
$$

$\widetilde{\mathbf{L}}_{2 n}$ and $\widetilde{\mathbf{L}}_{2 n+1}$ are linear functionals defined over vectors $\mathbf{u}$, and we shall say that a sequence $\tilde{\mathbf{L}}_{n}$ of such functionals is complete for a class $S$ of vectors if $\widetilde{\mathbf{L}}_{n}(\mathbf{u})=0 \quad(n=0,1, \ldots)$ implies $\mathbf{u}=0$. We have the following parallel to Theorem 1 .

THEOREM 8. The system (90') possesses a nontrivial solution of class $L^{2}(B)$ if and only if the set of functionals $\left\{\widetilde{\mathbf{L}}_{n}\right\}$ is incomplete for $L^{2}(B)$. If auxiliary conditions (91) are present, the set $\left\{\tilde{\mathbf{L}}_{n}\right\}$ must be augmented by the addition of $\left\{\mathbf{A}_{n}\right\}$.

It is now convenient to introduce the direct sum of $L^{2}(B)$ with itself:

$$
L_{2}^{2}(B)=L^{2}(B) \oplus L^{2}(B)
$$

This space consists of pairs

$$
\mathbf{u}=\left(u_{1}, u_{2}\right), u_{i} \in L^{2}(B) \text {. }
$$

Vector addition and scalar multiplication are defined by

$$
\mathbf{u}+\mathbf{v}=\left(u_{1}, u_{2}\right)+\left(v_{1}, v_{2}\right)=\left(u_{1}+v_{1}, u_{2}+v_{2}\right)
$$

and

$$
a \mathbf{u}=a\left(u_{1}, u_{2}\right)=\left(a u_{1}, a u_{2}\right)
$$

We introduce an inner product in $L_{2}^{2}(B)$ by means of 5

$$
\{\mathbf{u}, \mathbf{v}\}=\left\{\left(u_{1}, u_{2}\right),\left(v_{1}, v_{2}\right)\right\}=\int_{B}\left(u_{1} \bar{v}_{1}+u_{2} \bar{v}_{2}\right) d \omega,
$$

${ }^{5}$ In what follows, the parenthesis is used solely for the element pairs of $L_{2}^{2}(B)$. If the inner product in $L^{2}(B)$ is required, we shall write $(u, v)_{L^{2}(B)}$. 
and a norm by

$$
\begin{aligned}
\|\mathbf{u}\|^{2}= & \left\|\left(u_{1}, u_{2}\right)\right\|^{2}=\left\{\left(u_{1}, u_{2}\right),\left(u_{1}, u_{2}\right)\right\} \\
& \int_{B}\left(\left|u_{1}\right|^{2}+\left|u_{2}\right|^{2}\right) d \omega=\left\|u_{1}\right\|_{L^{2}(B)}^{2}+\left\|u_{2}\right\|_{L^{2}(B)}^{2} \cdot
\end{aligned}
$$

Under this norm, it is known that $L_{2}^{2}(B)$ becomes a Hilbert Space. By classical results, any bounded linear functional $\mathbf{T}$ over $L_{2}^{2}(B)$ possesses a representation of the form

$$
\mathbf{T}(\mathbf{u})=\{\mathbf{u}, \mathbf{v}\}=\int_{B}\left(u_{1} \bar{v}_{1}+u_{2} \bar{v}_{2}\right) d \omega
$$

for some $\mathbf{v} \in L_{2}^{2}(B)$. Hence we have the decomposition

$$
\mathbf{T}(\mathbf{u})=T_{1}\left(u_{1}\right)+T_{2}\left(u_{2}\right),
$$

where $T_{1}$ and $T_{2}$ are bounded linear functionals over $L^{2}(B)$. The converse evidently holds also. Moreover,

$$
\|\mathbf{T}\|^{2}=\|\mathbf{v}\|^{2}=\left\|v_{1}\right\|^{2}+\left\|v_{2}\right\|^{2}=\left\|T_{1}\right\|^{2}+\left\|T_{2}\right\|^{2}
$$

In what follows, we shall assume that $\tilde{\mathbf{L}}_{n}$ as well as $\mathbf{A}_{n}$ are linear and bounded over $L_{2}^{2}(B)$. The examples given in $\S 2$ are easily extended to the present case.

If $\left\{\mathbf{u}^{(n)}\right\}$ is a complete orthonormal system for $L^{2}(B)$, it may be shown by an extension of the usual proofs that each of the series $\sum_{n=0}^{\infty} u_{i}^{(n)}(z) \overline{\left[u_{j}^{(n)}(w)\right]}$ converges uniformly and absolutely in every closed bounded subdomain of $B \times B$. In addition, a strong Riesz-Fischer theorem exists for $L_{2}^{2}(B)$; that is, $\mathbf{u} \in L_{2}^{2}(B)$ if and only if

$$
\mathbf{u}=\sum_{n=0}^{\infty} a_{n} \mathbf{u}^{(n)} \text { with } \sum_{n=0}^{\infty}\left|a_{n}\right|^{2}<\propto
$$

and

$$
a_{n}=\left\{\mathbf{u}, \mathbf{u}^{(n)}\right\}
$$$$
(n=0, \cdots) \text {. }
$$

The convergence of each of the component series is uniform and absolute in every closed bounded subdomain of $B$. The array 
(98) $\mathscr{K}_{B}(Z ; \overline{\mathbb{W}})=\left(\begin{array}{cc}\sum_{n=0}^{\infty} u_{1}^{(n)}(z) \overline{u_{1}^{(n)}(w)} & \sum_{n=0}^{\infty} u_{1}^{(n)}(z) \overline{u_{2}^{(n)}(w)} \\ \sum_{n=0}^{\infty} u_{2}^{(n)}(z) \overline{u_{1}^{(n)}(w)} & \sum_{n=0}^{\infty} u_{2}^{(n)}(z) \overline{u_{2}^{(n)}(w)}\end{array}\right)$

will be known as a kernel tensor for the space $L_{2}^{2}(B)$. Each row of $\mathcal{\psi}_{B}$ is, for fixed $W \in B$, a vector element of $L_{2}^{2}(B)$ which we shall denote by $\mathcal{Z}_{B}^{1}(Z, \bar{W})$ and $\mathcal{K}_{B}^{(2)}(Z, \bar{W})$. Thus,

$$
\oiint_{B}(Z, \bar{W})=\left(\begin{array}{c}
\varkappa_{B}^{1}(Z ; \bar{W}) \\
\varkappa_{B}^{2}(Z ; \bar{W})
\end{array}\right) .
$$

If

$$
\mathbf{u}=\left(u_{1}, u_{2}\right) \in L_{2}^{2}(B)
$$

then we have

$$
u_{i}=\left\{\not{Z}_{B}^{i}(Z, \bar{W}), \overline{\mathbf{u}(w)}\right\}
$$

Let us consider, for example, the case $i=1$; then

$$
\mathbf{u}=\sum_{n=0}^{\infty} a_{n}\left(u_{1}^{(n)}, u_{2}^{(n)}\right)
$$

so that

$$
\begin{aligned}
& \left\{\mathscr{G}_{B}^{i}(Z ; W), \overline{\mathbf{u}(\mathbb{W})}\right\}=\left\{\left(\sum_{n=0}^{\infty} u_{1}^{(n)}(Z) \overline{u_{1}^{(n)}(\mathbb{W})}, \sum_{n=0}^{\infty} u_{1}^{(n)}(Z) \overline{u_{2}^{(n)}(\mathbb{W})}\right),\right. \\
& \left.\overline{\left(\sum_{n=0}^{\infty} a_{n} u_{1}^{(n)}(\mathbb{W}), \sum_{n=0}^{\infty} a_{n} u_{2}^{(n)}(\mathbb{W})\right)}\right\} \\
& =\sum_{\substack{m=0 \\
n=0}}^{\infty} a_{m} u_{1}^{(n)}(Z) \int_{B} u_{1}^{(m)}(\mathbb{W}) \overline{u_{1}^{(n)}(\mathbb{W})}+u_{2}^{(m)}(\mathbb{W}) \overline{u_{2}^{(n)}(\mathbb{W})} d \omega,
\end{aligned}
$$


which by orthonormality reduces to

$$
\sum_{n=0}^{\infty} a_{n} u_{1}^{(n)}(Z)=u_{1}(Z)
$$

The reproducing property may be written more compactly as

$$
\mathbf{u}(Z)=\left\{\mathbf{u}(\mathbb{W}), \mathcal{K}_{B}(\mathbb{W} ; \bar{Z})\right\} .
$$

It can be seen that if $\left\{u_{n}\right\}$ is a complete orthonormal system for $L^{2}(B)$, then the set of vectors

$$
\begin{aligned}
& \mathbf{u}_{2 n}=\left(u_{n}, 0\right) \\
& (n=0,1, \cdots), \\
& \mathbf{u}_{2 n+1}=\left(0, u_{n}\right) \\
& (n=0,1, \cdots) \text {, }
\end{aligned}
$$

is complete and orthonormal for $L_{2}^{2}(B)$. With this special selection, we find a kernel tensor of the form

$$
K_{B}(Z ; W)=\left(\begin{array}{cc}
K_{B}(Z ; \bar{W}) & 0 \\
0 & K_{B}(Z, \bar{W})
\end{array}\right) \text {, }
$$

where $K_{B}$ is the kernel for $L^{2}(B)$.

We come now to the analogue of Theorem 2 .

THEOREM 9. Let $\left\{\tilde{\mathbf{L}}_{k}\right\}$ be a set of linear functionals each of which is defined and bounded over $L_{2}^{2}(B)$. The set $\left\{\tilde{\mathbf{L}}_{k}\right\}$ will be assumed independent. Then there exists a set of pairs

$$
\varnothing_{k}^{*}(Z)=\left(\phi_{k, 1}^{*}, \phi_{k, 2}^{*}\right) \quad(k=0,1, \ldots)
$$

and a set of linear functionals $\left\{\tilde{\mathbf{L}}_{k}^{*}\right\} \quad(n=0,1, \cdots)$ which possess the following properties:

(a) Each $\varnothing_{k}^{*}$ is of class $L_{2}^{2}(B)$, and the set is orthonormal:

$$
\left\{\varnothing_{k}^{*}, \varnothing_{j}^{*}\right\}=\delta_{j k}
$$

(b) Each $\widetilde{\mathbf{L}}_{k}^{*}$ is a finite linear combination of the functionals $\tilde{\mathbf{L}}_{k}$ : 


$$
\widetilde{\mathbf{L}}_{k}^{*}=\sum_{p=0}^{k} a_{k p} \widetilde{\mathbf{L}}_{p}
$$

for an appropriate set of constants $a_{k p}$.

(c) The sets $\left\{\tilde{\mathbf{L}}_{k}^{*}\right\}$ and $\left\{\varnothing_{k}^{*}\right\}$ are biorthonormal:

$$
\widetilde{\mathbf{L}}_{j}^{*}\left(\varnothing_{k}^{*}\right)=\delta_{j k},
$$

(d) For all $\mathbf{v} \in L_{2}^{2}(B)$ we have

$$
\widetilde{\mathbf{L}}_{k}^{*}(\mathbf{V})=\left\{\mathbf{v}, \varnothing_{k}^{*}\right\} .
$$

(e) The pairs $\varnothing_{p}^{*}$ may be obtained by taking the set

$$
\phi_{n}(Z)=\tilde{\mathbf{L}}_{n, \bar{w}} \varkappa_{B}(Z, \bar{W})
$$$$
(n=0,1, \ldots)
$$

and orthonormalizing them by the Gram-Schmidt process.

(f) The set $\left\{\varnothing_{k}\right\}$ (or $\left\{\varnothing_{k}^{*}\right\}$ ) is complete for $L_{2}^{2}(B)$ if and only if the set $\left\{\tilde{\mathbf{L}}_{k}\right\}$ is complete for $L_{2}^{2}(B)$.

By (107) is meant that

$$
\phi_{n, i}(Z)=\widetilde{L}_{n, \bar{w}} \mathcal{\not}_{B}^{(i)}(Z ; \bar{W})
$$

where

$$
\varnothing_{n}(Z)=\left(\phi_{n, 1}(Z), \phi_{n, 2}(Z)\right)
$$

Using the specific set of functionals (92), we construct the related biorthonormal sets $\left\{\tilde{\mathbf{L}}_{k}^{*}\right\}$ and $\left\{\varnothing_{k}^{*}\right\}$. We then have the following analogue of Theorem 3 .

THEOREM 10. The vector $\mathbf{g}(z)$ is a solution of the system (90) of class $L_{2}^{2}(B)$ if and only if there exists an $\mathbf{f}(Z) \in L_{2}^{2}(B)$ for which

$$
\mathbf{g}(Z)=\mathbf{f}(Z)-\sum_{k=0}^{\infty} \tilde{\mathbf{L}}_{k}^{*}(\mathbf{f}) \varnothing_{k}^{*}(Z)
$$

For each $\mathbf{f} \in L_{2}^{2}(B)$, the series in (109) converges uniformly and absolutely in every closed bounded subdomain of $B$. It is simultaneously a Fourier series and an interpolation series whose terms may be obtained by interpolating to $f$ 
by means of $\left\{\widetilde{\mathbf{L}}_{k}\right\}$.

\section{REFERENCES}

1. N. Aronszajn, Theory of reproducing kernels, Trans. Amer. Math. Soc. (1950), $337-404$.

2. S. Banach, Théorie des operations linéaires, Warsaw, 1933.

3. S. Bergman, Zur Theorie der Funktionen, die eine lineare partielle Differentialgleichung befriedigen, Recueil Mathematique, nouv. ser. 2 (44) (1937), 1169-1198.

4. - Zur Theorie der Linearen Integral-und Funktionalgleichungen im Komplexen Gebiet, Bulletin de l'Institute de Mathématiques et Mécanique à l'Université Koubycheff de Tomsk 1 (1937), 242-256.

5. vol. 5, New York, 1950. 1953.

6. S. Bergman and M. Schiffer, Kernel functions in mathematical physics, New York,

7. P. Davis, On the applicability of linear differential operators of infinite order to functions of class $L^{2}(B)$, Amer. J. Math. 74 (1952), 475-491.

8. P. Davis and J. L. Walsh, Representations and extensions of bounded linear functionals defined on classes of analytic functions, Trans. Amer. Math. Soc. 76 (1954), $190-206$.

9. P. Davis and H. Pollak, Linear functionals and analytic continuation problems, Pacific J. Math. 3 (1953), 47-72.

10. W. F. Osgood, Funktionentheorie, Bd. III, Leipzig-Berlin, 1927. 1913.

11. F. Riesz, Les systèmes d'equations linéaires à une infinite'd'inconnues, Paris,

12. J. L. Walsh and P.Davis, Interpolation and orthonormal systems, J. Analyse Math. 2 (1952), 1- 28.

13. A. Wintner, A criterion for the non-existence of $L^{2}$ solutions of a non-oscillating differential equation, London J. Math. 25 (1950), 347-351.

\section{NATIONAL BUREAU OF STANDARDS}

WASHINGTON, D.C. 


\section{PACIFIC JOURNAL OF MATHEMATICS}

\section{EDITORS}

\author{
M.M. SCHIFFER * \\ Stanford University \\ Stanford, California \\ E. HEWITT \\ University of Washington \\ Seattle 5, Washington
}

\section{R.P. DILWORTH}

California Institute of Technology Pasadena 4, California

\section{E. F. BECKENBACH**}

University of California

Los Angeles 24, California

\section{ASSOCIATE EDITORS}

$\begin{array}{llll}\text { H. BUSEMANN } & \text { P.R. HALMOS } & \text { BØRGE JESSEN } & \text { J. J. STOKER } \\ \text { HERBERT FEDERER } & \text { HEINZ HOPF } & \text { PAUL LÉVY } & \text { E.G. STRAUS } \\ \text { MARSHALL HALL } & \text { R.D. JAMES } & \text { GEORGE PÓLYA } & \text { KÔSAKU YOSIDA }\end{array}$

\section{SPONSORS}

UNIVERSITY OF BRITISH COLUMBIA

CALIFORNIA INSTITUTE OF TECHNOLOGY

UNIVERSITY OF CALIFORNIA, BERKELEY

UNIVERSITY OF CALIFORNIA, DAVIS

UNIVERSITY OF CALIFORNIA, LOS ANGELES

UNIVERSITY OF CALIFORNIA, SANTA BARBARA

UNIVERSITY OF NEVADA

OREGON STATE COLLEGE

UNIVERSITY OF OREGON
UNIVERSITY OF SOUTHERN CALIFORNIA STANFORD RESEARCH INSTITUTE STANFORD UNIVERSITY WASHINGTON STATE COLLEGE UNIVERSITY OF WASHINGTON

AMERICAN MATHEMATICAL SOCIETY HUGHES AIRCRAFT COMPANY

Mathematical papers intended for publication in the Pacific Journal of Mathematics should be typewritten (double spaced), and the author should keep a complete copy. Manuscripts may be sent to any of the editors. Manuscripts intended for the outgoing editors should be sent to their successors. All other communications to the editors should be addressed to the managing editor, E.G. Straus, at the University of California Los Angeles 24, California.

50 reprints of each article are furnished free of charge; additional copies may be obtained at cost in multiples of 50 .

The Pacific Journal of Mathematics is published quarterly, in March, June, September, and December. The price per volume (4 numbers) is $\$ 12.00$; single issues, $\$ 3.50$; back numbers (Volumes $1,2,3$ ) are available at $\$ 2.50$ per copy. Special price to individual faculty members of supporting institutions and to individual members of the American Mathematical Society: $\$ 4.00$ per volume; single issues, $\$ 1.25$.

Subscriptions, orders for back numbers, and changes of address should be sent to the publishers, University of California Press, ,Berkeley 4, California.

Printed at Ann Arbor, Michigan. Entered as second class matter at the Post Office, Berkeley, California.

* To be succeeded in 1955, by H.L. Royden, Stanford University, Stanford, California.

** To be succeeded in 1955, by E.G. Straus, University of California, Los Angeles 24, Calif.

UNIVERSITY OF CALIFORNIA PRESS - BERKELEY AND LOS ANGELES 


\section{Pacific Journal of Mathematics}

\section{Vol. 4, No. $4 \quad$ August, 1954}

Paul Civin, Orthonormal cyclic groups .................... 481

Kenneth Lloyd Cooke, The rate of increase of real continuous solutions of

algebraic differential-difference equations of the first order ......... 483

Philip J. Davis, Linear functional equations and interpolation series . . . . . 503

F. Herzog and G. Piranian, Sets of radial continuity of analytic functions . . 533

P. C. Rosenbloom, Comments on the preceding paper by Herzog and

Piranian ............................................. 539

Donald G. Higman, Remarks on splitting extensions .............. 545

Margaret Jackson, Transformations of series of the type ${ }_{3} \Psi_{3} \ldots \ldots \ldots \ldots 557$

Herman Rubin and Patrick Colonel Suppes, Transformations of systems of relativistic particle mechanics ....................... 563

A. Seidenberg, On the dimension theory of rings. II .............. 603

Bertram Yood, Difference algebras of linear transformations on a Banach

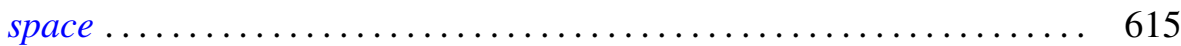

